\title{
Supersaturation calculation in large eddy simulation models for prediction of the droplet number concentration
}

\author{
O. Thouron ${ }^{1}$, J.-L. Brenguier ${ }^{2}$, and F. Burnet ${ }^{2}$ \\ ${ }^{1}$ CERFACS/A\&E, URA1875, 42 avenue Gaspard Coriolis, 31057 Toulouse CEDEX 01, France \\ ${ }^{2}$ CNRM/GMEI/MNPCA, METEOFRANCE, URA1357, 42 avenue Gaspard Coriolis, 31057 Toulouse CEDEX 01, France
}

Correspondence to: O. Thouron (odile.thouron@cerfacs.fr)

Received: 24 October 2011 - Published in Geosci. Model Dev. Discuss.: 5 December 2011

Revised: 25 April 2012 - Accepted: 27 April 2012 - Published: 23 May 2012

\begin{abstract}
A new parameterization scheme is described for calculation of supersaturation in LES models that specifically aims at the simulation of cloud condensation nuclei (CCN) activation and prediction of the droplet number concentration. The scheme is tested against current parameterizations in the framework of the Meso-NH LES model. It is shown that the saturation adjustment scheme, based on parameterizations of $\mathrm{CCN}$ activation in a convective updraft, overestimates the droplet concentration in the cloud core, while it cannot simulate cloud top supersaturation production due to mixing between cloudy and clear air. A supersaturation diagnostic scheme mitigates these artefacts by accounting for the presence of already condensed water in the cloud core, but it is too sensitive to supersaturation fluctuations at cloud top and produces spurious $\mathrm{CCN}$ activation during cloud top mixing. The proposed pseudo-prognostic scheme shows performance similar to the diagnostic one in the cloud core but significantly mitigates CCN activation at cloud top.
\end{abstract}

\section{Introduction}

Equivalent liquid potential temperature and the total water mixing ratio, $r_{\mathrm{t}}$, are two conservative quantities in atmospheric numerical models. In a cloudy atmosphere, $r_{\mathrm{t}}$ is distributed onto water vapour and condensed water, either liquid or ice. This paper is focused on liquid water clouds, also referred to as warm clouds, and the liquid water mixing ratio is designated by $r_{\mathrm{c}}$.

The water vapour mixing ratio, $r_{\mathrm{v}}$, is thus equal to $r_{\mathrm{t}}-r_{\mathrm{c}}$, and the saturation water mixing ratio $r_{\mathrm{S}}$ can be derived from pressure and temperature:
$r_{\mathrm{S}}(P, T)=\varepsilon \frac{e_{\mathrm{S}}(T)}{P-e_{\mathrm{S}}(T)}$

where $e_{\mathrm{S}}(T)$ is the saturation water vapour pressure over an infinite plane of pure water, $T$ is the absolute temperature, and $P$ is the pressure and $\varepsilon$ is the ratio of the molecular weight of water vapour to dry air (Pruppacher and Klett, 1997).

The supersaturation, $S$, expresses the relative deviation of the water vapour mixing ratio with respect to its saturation value:

$S=\left(\frac{r_{\mathrm{V}}}{r_{\mathrm{s}}}-1\right)$.

Supersaturation, which is currently expressed in $\%(S \times$ $100)$, rarely exceeds a few percents in warm clouds, because there are generally enough cloud condensation nuclei and droplets to deplete water vapour excess. Consequently, adjustment to saturation is a good approximation, to within a few percents, for a diagnostic of the liquid water mixing ratio in warm clouds.

$r_{\mathrm{c}}=r_{\mathrm{t}}-r_{\mathrm{s}}$

Calculation of supersaturation, however, is still required when the model also aims at predicting the number concentration of cloud droplets. Indeed, droplets form on cloud condensation nuclei, CCNs, that are hydrophilic, partly soluble particles at the surface of which the saturation water vapour pressure is lower than $e_{\mathrm{S}}(T)$. Due to surface tension effects, however, the vapour pressure at the surface of a pure water particle increases when the particle size decreases. Because of this competition between solute and surface tension 
effects, the water vapour pressure at the surface of a particle exhibits a maximum when expressed as a function of the wet particle size. CCNs have to experience a supersaturation greater than this maximum to be activated as cloud droplets. Hence, a population of CCNs is currently represented by its activation spectrum, $N_{\text {act }}(S)$, that establishes a direct link between the ambient supersaturation, $S$, and the number concentration of activated particles, $N_{\text {act }}$.

In summary, the calculation of supersaturation in a cloudy atmosphere is not necessary to derive the liquid water mixing ratio, as long as an uncertainty of a few percents is acceptable, but its peak value shall be determined when the model also aims at predicting the cloud droplet number concentration, $N_{\mathrm{c}}$ (Pruppacher and Klett, 1997).

Warm cloud microphysics schemes have thus been developed assuming saturation adjustment $(S=0)$ and using a diagnostic scheme for deriving the peak supersaturation (Twomey, 1959; Ghan et al., 1995; Cohard et al., 1998). Such an approximation was quite satisfactory when the vertical resolution was greater than $100 \mathrm{~m}$ and the time steps longer than a few tens of seconds, which is currently how long it takes for supersaturation to increase at the base of a cloud updraft, reach its maximum, typically $50 \mathrm{~m}$ above the base, and fall down to its pseudo-equilibrium value.

Today, LES models of cloud microphysics are run with a vertical resolution of less than $10 \mathrm{~m}$ and time steps of less than $1 \mathrm{~s}$. This is not fine enough for a complete prognostic calculation of supersaturation that requires time steps of milliseconds, typically, but this is too thin and too fast for assuming that supersaturation reaches and passes its maximum within a time step.

Consequently, new parameterizations have been developed to explicitly calculate supersaturation and simulate $\mathrm{CCN}$ activation and droplet condensation/evaporation. However, a numerical artefact currently happens at the interface between a cloud and its environment, when cloudy air is progressively advected in a clear grid box (Clark, 1973; Klaassen and Clark, 1985; Grabowski, 1989; Grabowski and Smolarkiewicz, 1990; Kogan et al., 1995; Stevens et al., 1996a; Grabowski and Morrison, 2008), for instance at the top of a stratoculumus layer. Indeed, explicit calculation of supersaturation is problematic, because it is a second order variable compared to the model prognostic variables $T$ and $r_{\mathrm{t}}$. Small numerical errors in the advection scheme for instance can result in large fluctuations of the derived supersaturation. This artefact generates spurious supersaturation peak values, hence $\mathrm{CCN}$ activation and results in unrealistic $N_{\mathrm{c}}$ values at cloud top.

A new scheme is proposed here to reduce these artefacts. Parameterization schemes are briefly described in the next section, starting with the two commonly used techniques, adjustment to saturation (Sect. 2.1) and saturation diagnostic (Sect. 2.2), before describing the proposed approach in Sect. 2.3. The three schemes are then tested in a 3-D frame- work of an LES model (Sect. 3) to evaluate the benefits of the proposed parameterization.

\section{Parameterization schemes}

To simulate warm clouds, including prediction of the cloud droplet number concentration, three prognostic variables are required beyond dynamics: the equivalent liquid water potential temperature $\theta_{1}$ or any thermodynamical variable conserved during condensation/evaporation processes, the total water mixing ratio $r_{\mathrm{t}}$ and the cloud droplet number concentration $N_{\mathrm{c}}$. Note that additional variables are also required to simulate the formation of precipitation, such as the precipitating water mixing ratio and precipitating particle number concentration (Khairoutdinov and Kogan, 2000).

The parameterization schemes described in this section aim at the prediction of the cloud water mixing ratio $r_{\mathrm{c}}$ and of the number concentration of activated CCNs. A key issue is to avoid activation of already activated CCNs each time supersaturation is produced in a model grid box. This is only feasible if additional prognostic variables are used for describing the $\mathrm{CCN}$ population in the 3-D framework, including a sink term reflecting $\mathrm{CCN}$ activation.

When such a CCN scheme is not operational and the $\mathrm{CCN}$ population is assumed uniform over the whole domain, the problem can be partially addressed with only one additional prognostic variable. Indeed, beside advection, the cloud droplet number concentration increases when new CCNs are activated and decreases when droplets totally evaporate. At this stage, there is a direct correspondence between the activated CCN number concentration and the cloud droplet number concentration. The cloud droplet number concentration, however, also decreases when droplets collide, either with other droplets (autoconversion) or with precipitating drops (accretion). The so-called collection process is not conservative for the number concentration of activated CCNs. It is therefore common to use an additional prognostic variable $N_{\text {act }}$ to represent the number concentration of activated CCNs (Cohard et al., 1998). Like $N_{\mathrm{c}}, N_{\text {act }}$ is driven by $\mathrm{CCN}$ activation and droplet evaporation but it is not affected by the collection process.

\subsection{Scheme A: adjustment to saturation with parameterized peak supersaturation}

In this scheme, the cloud water mixing ratio is a diagnostic variable that is directly derived at each time step from total water, pressure and temperature (3), assuming no supersaturation. To diagnose the number concentration of activated $\mathrm{CCNs}$, the supersaturation peak value, $S_{\max }$, is derived from the time evolution equation of supersaturation in an ascending adiabatic volume of air, by setting the time derivative of supersaturation to 0 at the maximum: 
$\frac{d S}{\mathrm{~d} t}=\frac{1}{r_{\mathrm{s}}} \frac{\mathrm{d} r_{\mathrm{v}}}{\mathrm{d} t}-\frac{\left(S_{\max }+1\right)}{r_{\mathrm{s}}(P, T)} \frac{\mathrm{d} r_{\mathrm{s}}}{\mathrm{d} t}=0$.

$\frac{\mathrm{d} r_{\mathrm{s}}}{\mathrm{d} t}=\frac{\mathrm{d} r_{\mathrm{s}}}{\mathrm{d} T} \frac{\mathrm{d} T}{\mathrm{~d} t}$ expresses a forcing due to cooling by adiabatic ascent and $\frac{\mathrm{d} r_{\mathrm{v}}}{\mathrm{d} t}$ expresses the sink of water vapour condensed on droplets that depend on the CCN spectrum. Various formulae have been derived to solve the system of differential equations using a parameterized description of the $\mathrm{CCN}$ activation spectrum (Leaitch et al., 1986; Ghan et al., 1995; Cohard et al., 1998; Abdul-Razzak and Ghan; 2000).

The diagnostic of maximum supersaturation, $S_{\max }$, is directly translated into a diagnostic of activated $\mathrm{CCN}$ number concentration, $N_{\text {act }}$, using the CCN activation spectrum. To account for the non-reversibility of the activation process, $N_{\text {act }}$ and $N_{\mathrm{c}}$ are updated each time the $N_{\text {act }}\left(S_{\max }\right)$ diagnosed value is higher than the current one. This scheme is the currently implemented in the Méso-NH research model.

Because the forcing is expressed as a function of vertical velocity only, these formulae are well suited for $\mathrm{CCN}$ activation at the base of convective clouds, but they shall be adapted when activation is mainly driven for instance by radiative cooling. A second limitation is that the formulae hold for grid boxes with no initial liquid water content, while any pre-existing condensed water in a model grid box significantly reduces the supersaturation peak value.

The main limitation, though, is that the solution corresponds to the maximum supersaturation when the forcing is maintained until the maximum is reached. With a vertical velocity of the order of $1 \mathrm{~m} \mathrm{~s}^{-1}$ and a typical CCN distribution, the maximum is reached after a few tens of seconds while the convective cell has already travelled a few tens of meters. These formulae are therefore not suited for fine spatial and time resolution simulations of less than $10 \mathrm{~m}$ and $10 \mathrm{~s}$, respectively.

\subsection{Scheme B: diagnostic of supersaturation}

With an additional prognostic variable, either $r_{\mathrm{v}}$ or $r_{\mathrm{c}}$, it becomes possible to directly diagnose supersaturation. In a first step, supersaturation is derived from the values of the thermodynamical prognostic variables $\theta_{\mathrm{l}}, r_{\mathrm{t}}$ and $r_{\mathrm{c}}\left(\right.$ or $r_{\mathrm{v}}$ ) after advection and modification by processes other than condensation/evaporation:

$S^{\prime}=\frac{r_{\mathrm{t}}-r_{\mathrm{c}}-r_{\mathrm{s}}}{r_{\mathrm{s}}}$

or

$S^{\prime}=\frac{r_{\mathrm{v}}-r_{\mathrm{s}}}{r_{\mathrm{s}}}$.

This $S^{\prime}$ value is then used for both CCN activation (when $\left.N_{\mathrm{CCN}}\left(S^{\prime}\right)>N_{\text {act }}\right)$ and droplet growth/evaporation using $\frac{\mathrm{d} r_{\mathrm{c}}}{\mathrm{d} t}=4 \pi \rho_{\mathrm{w}} G S^{\prime} I$

where $I$ is the integral radius of the droplet size distribution, and $G=\frac{1}{F_{k}+F_{D}}$ where $F_{k}$ is a function of thermal conductivity of the air and $F_{D}$ is a function of the diffusivity of water vapour (Pruppacher et Klett, 1997). The two conservative variables $\theta_{\mathrm{l}}$ and $r_{\mathrm{t}}$ are not affected by condensation/evaporation, but the final value of $r_{\mathrm{c}}$ (or $r_{\mathrm{v}}$ ) shall be updated according to Eq. (7).

The initial diagnostic of supersaturation provided by Eq. (6) is very sensitive to small errors in $\theta_{\mathrm{l}}, r_{\mathrm{t}}$ and $r_{\mathrm{c}}$ or $r_{\mathrm{v}}$ because it is a second order variable. Indeed, supersaturation rarely exceeds a few percents. The system is well-buffered for condensation/evaporation, since over(under)estimated supersaturation leads to an over(under)estimation of the condensation rate, so that, on average, $r_{\mathrm{c}}$ remains close to its optimal value. Spurious positive peak values of the supersaturation, however, have a significant impact on CCN activation because the process is non-reversible, hence producing unexpected high values of the cloud droplet number concentration.

\subsection{Scheme C: pseudo-prognostic of supersaturation}

The scheme proposed here aims at mitigating the weaknesses of the two approaches described above, when the time step and vertical resolution of the model get shorter than $10 \mathrm{~s}$ and $10 \mathrm{~m}$, respectively. A parameterization of the supersaturation peak value, such as the ones discussed in Sect. 2.1, is precluded because the peak value cannot be reached during so short time steps. Diagnostic of supersaturation is also precluded, because it amplifies small errors in the advection of heat and moisture. The objective is also to derive an estimate of the supersaturation that combines both the forcing by the thermodynamics and the sink/source by condensation/evaporation of pre-existing droplets.

Like in Sect. 2.2, the scheme requires an additional prognostic variable for the condensate and a semi-prognostic variable to communicate the estimate of supersaturation from one time step to the next one, referred here to as $S^{t}$. The forcing term is derived from the values of the thermodynamical prognostic variables $\theta_{\mathrm{l}}, r_{\mathrm{t}}$ and $r_{\mathrm{c}}$ (or $\left.r_{\mathrm{v}}\right)$ after advection and modification by processes other than condensation/evaporation:

$\left(\frac{d S}{\mathrm{~d} t}\right)_{\mathrm{f}}=\frac{S^{\prime}-S^{t}}{\Delta t}$

where $\Delta t$ is the model time step and $S^{\prime}$ is calculated using Eq. (6) (as in Sect. 2.2). The condensation/evaporation term $\left(\frac{d S}{\mathrm{~d} t}\right)_{\mathrm{ce}}$ is derived (see Appendix A): 


$$
\left(\frac{d S}{\mathrm{~d} t}\right)_{\mathrm{ce}}=-\left(1+\left(S^{t}+1\right) \frac{L_{\mathrm{v}}}{C_{\mathrm{p}}} \frac{\mathrm{d} r_{\mathrm{s}}}{\mathrm{d} T}\right) \frac{1}{r_{\mathrm{s}}} \frac{\mathrm{d} r_{\mathrm{c}}}{\mathrm{d} t}
$$

where $\frac{\mathrm{d} r_{c}}{\mathrm{~d} t}$ is calculated using $S^{t}$ instead of $S^{\prime}$ in Eq. (7). The final value of supersaturation is then derived:

$S^{t+1}=S^{t}+\left(\left(\frac{d S}{\mathrm{~d} t}\right)_{f}+\left(\frac{d S}{\mathrm{~d} t}\right)_{\mathrm{ce}}\right) \Delta t$.

$S^{t}$ is used for CCN activation (when $N_{\mathrm{CCN}}\left(S^{t}\right)>N_{\text {act }}$ ). $S^{t+1}$ is stored for the next time step.

\section{Tests in a 3-D framework}

\subsection{Model setup}

To evaluate the three techniques discussed above, the mesoscale nonhydrostatic atmospheric research model (Meso-NH) was used. This model was jointly developed by Météo-France Centre National de Recherches Meteorologiques (CNRM) and Laboratoire d'Aérologie, for largeto small-scale simulations of atmospheric phenomena. The dynamical core of the model (Lafore et al., 1998) was completed by a 3-D one-and-a-half turbulence scheme based on a prognostic equation of kinetic energy (Cuxart et al., 2000), with a Deardorff mixing length. Surface fluxes were computed using the Charnock's relation for roughness length (Charnock, 1955). Shortwave and longwave radiative transfer calculations were performed following the European Centre for Medium-Range Weather Forecasts' (ECMWF) Fouquart and Morcrette formulation (Morcrette, 1991). The model includes a two-moment bulk microphysical scheme based on the parameterization of Khairoutdinov and Kogan (2000), which was specifically designed for LES studies of warm stratocumulus clouds (Geoffroy et al., 2008). A complete description of the model can be found online at http://www.aero.obs-mip.fr/mesonh/index2. html. This model has been extensively used for LES studies of boundary layer clouds, with the one moment and two moments microphysical schemes (Chosson, 2007; Geoffroy, 2008; Sandu, 2008, 2009).

The model was used here with a horizontal resolution of $50 \mathrm{~m}$ and a vertical resolution varying from 50 to $10 \mathrm{~m}$, with the finest resolution in the cloud and at the inversion layer. Periodic boundary conditions were applied horizontally, and the top of the domain was at $1.5-\mathrm{km}$. The time step was set to $1 \mathrm{~s}$ and the ECMWF radiation scheme was called every second in cloudy columns and every $120 \mathrm{~s}$ in clear columns. To avoid interactions between the cloud structures and the domain size, the horizontal domain dimension was set to $10 \mathrm{~km}$ for a 3-h simulation time run, following de De Roode et al. (2004). The prognostic variables were $\theta_{\mathrm{l}}, r_{\mathrm{t}}, r_{\mathrm{c}}$, and $N_{\mathrm{c}}$.

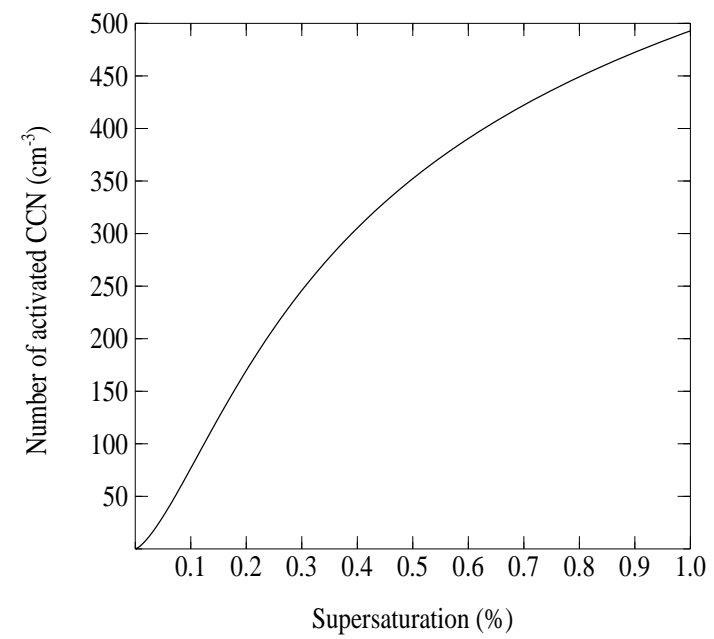

Fig. 1. Number of activated $\mathrm{CCN}$ in function of the supersaturation obtained from Cohard et al. (2000) for the continental case, with concentration of activated $\mathrm{CCN}$ at a very high (infinite) supersaturation of $1236 \mathrm{~cm}^{-3}$.

The model initialisation fields originated from a nonprecipitating stratocumulus cloud layer case that was observed on 9 July 1997, over the northeast Atlantic, north of the Canary Islands, during the ACE-2 Cloudy Column experiment (Brenguier et al., 2000a). The inversion layer was located at $960 \mathrm{~m}$, with sharp jumps in both water vapour mixing ratio and liquid water equivalent temperature. To reduce the spin-up time, the simulations were performed with a subsaturated initial profile and a cooling was applied during the first hour of simulation. The simulation was then continued for two more hours after cooling had been shutoff. Unlike the observed case, nighttime conditions were assumed.

Following Cohard et al. (2000), aerosol particles were assumed to be $\log$ normally distributed. The $\mathrm{CCN}$ activation spectrum is prescribed:

$N_{\mathrm{CCN}}=C S^{k} F\left(\mu, \frac{k}{2}, \frac{k}{2}+1, \beta S^{2}\right)$

where $S(\%)$ is supersaturation and $F(a, b, c, x)$ is the hypergeometric function (Press et al., 1992). $C, k, \mu$ and $\beta$ are activation spectrum coefficients that can be tuned to represent various aerosol types. $C, k, \mu$ and $\beta$ are set to $1800.10^{3} \mathrm{~cm}^{3}$, $1.403,25.499$ and 0.834 , respectively. These values are typical of a continental case, in which aerosol particles are composed of $\left(\mathrm{NH}_{4}\right)_{2} \mathrm{SO}_{4}$, with a concentration of activated $\mathrm{CCN}$ at $1 \%$ supersaturation of $500 \mathrm{~cm}^{-3}$, as shown in Fig. 1.

To better evaluate the performance of the parameterizations and their sensitivity to entrainment-mixing processes, two simulations were performed with the same initialisation fields except for the values of the total water mixing ratio in the free troposphere equal to $5 \mathrm{~g} \mathrm{~kg}^{-1}$ and $8 \mathrm{~g} \mathrm{~kg}^{-1}$, respectively. These are thus referred to as the DRY and WET case. 


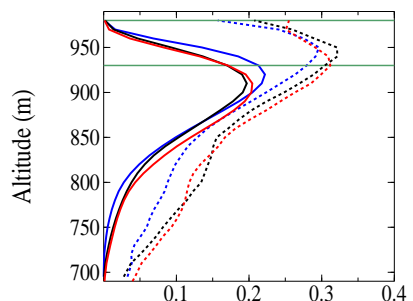

Liquid water content (g.m $\left.\mathrm{m}^{-3}\right)$
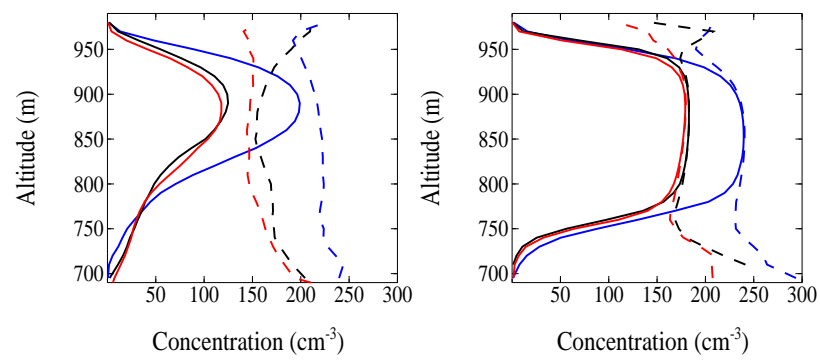

Fig. 2. Vertical profiles of LWC (top) and cloud droplet number concentration (bottom) averaged over $N_{\max }$ (Solid line) and over $N(k)$ (dashed line) for the DRY (left) and WET (right) case. Blue, black and red correspond to the "adjustment" or A, "diagnostic" or $\mathrm{B}$, and "pseudo-prognostic" or C schemes, respectively. The upper $100 \mathrm{~m}$ of the cloud layer is represented by the two green bars.

\subsection{General features}

Figure 2 shows the vertical profiles of liquid water content, $q_{\mathrm{c}}$, (top) and cloud droplet number concentration, $N_{\mathrm{c}}$, (bottom) for the DRY (left) and WET (right) simulations. $q_{\mathrm{c}}$ is used here instead of mixing ratio, $r_{\mathrm{c}}$, to facilitate the comparison with in situ observations. The dashed lines represent values averaged over the number of cloudy cells at each level $k$, denoted $N(k)$, while solid lines represent values averaged over domain cloud fraction $N_{\max }$ equal to $\max (N(k))$. Blue, black and red correspond to the "adjustment" or A, "diagnostic" or B, and "pseudo-prognostic" or C schemes, respectively.

The top row suggests that the schemes have no significant impact on the LWC profiles, although the A scheme exhibits a slightly greater and higher maximum in the DRY case. The DRY case also shows noticeable differences between the domain and layer cloud fraction averages that reflect the presence of clear air patches due to entrainment of very dry air from above the inversion.

In contrast, the cloud droplet concentration profiles (bottom) reveal that the A scheme significantly overestimates $N_{\mathrm{c}}$. The dashed lines, which emphasize the differences at cloud base and top, where the layer cloud fraction is reduced, reveal that the overestimation starts at cloud base during activation of $\mathrm{CCN}$ in updraft.

The B and C schemes produce similar $N_{\mathrm{c}}$ predictions, except at cloud top where the B scheme starts overestimating the cloud droplet number concentration and generates $N_{\mathrm{c}}$ values as high as the A scheme in both the DRY and WET simulations. This feature reflects the final statement in Sect. 2.2 about the sensitivity of the diagnostic scheme to advection errors at the interface between cloudy and clear air.

\subsection{CCN activation}

To better understand these two specific features, widespread overestimation of cloud droplet number concentration from base to top with the A scheme and at the top only with the B scheme, it is useful to explore the activation process in depth. Figure 3 for the DRY case shows(from top to bottom) the results obtained with the A, B and C schemes, respectively. The left column is for the vertical distribution of supersaturation values in model grid boxes where $\mathrm{CCN}$ activation occurs. The middle column shows the number concentration of activated particles during the time step, and the right column represents the resulting number concentration of cloud droplets in all cloudy grid boxes. The far left graph shows the PDF of occurrence of activation events in the vertical, while the ones under each figure are for the PDF of parameter values. For comparison, the outcomes of the three schemes are superimposed in each PDF graph with the current one in bold.

This figure reveals that the A scheme produces supersaturation values greater than the two others and, at the middle of the cloud, values greater than at cloud base. This directly reflects the fact that the A scheme relies on a parameterization of the $\mathrm{CCN}$ activation process that only accounts for the vertical velocity and does not consider the sink term due to existing LWC. In contrast, the B and C schemes that account for the presence of LWC in a grid box generate lower supersaturation values, with greater ones at cloud base. Moreover, the A scheme also assumes that the supersaturation produced during a single time step lasts long enough for the $\mathrm{CCN}$ activation to be completed. Because supersaturation is a noisy parameter, the chances to activate large concentrations of $\mathrm{CCN}$ each time it reaches its maximum are significantly increased. In contrast, the $\mathrm{B}$ and $\mathrm{C}$ schemes progressively activate small amounts of $\mathrm{CCN}\left(<40 \mathrm{~cm}^{-3}\right)$, as simulated for instance when using a high resolution explicit supersaturation scheme in a 1-D framework, although both schemes build up cloud droplet number concentration values of about $150 \mathrm{~cm}^{-3}$ and a few values greater than $200 \mathrm{~cm}^{-3}$.

There is however a noticeable difference between the $\mathrm{B}$ and $\mathrm{C}$ schemes, namely that the $\mathrm{B}$ scheme only produces spurious peak values of supersaturation at cloud top, hence spurious activation of new CCNs and spurious values of cloud droplet number concentration at cloud top. These high concentrations of activated CCNs are reflected in the corresponding PDF (middle column) that shows similar probabilities of high concentrations of activated CCNs for the A and $\mathrm{B}$ schemes (black and blue curves), while such occurrences fall below $10^{-4}$ with the $\mathrm{C}$ scheme (red curve) when the concentration of activated CCNs becomes greater than $60 \mathrm{~cm}^{-3}$. 

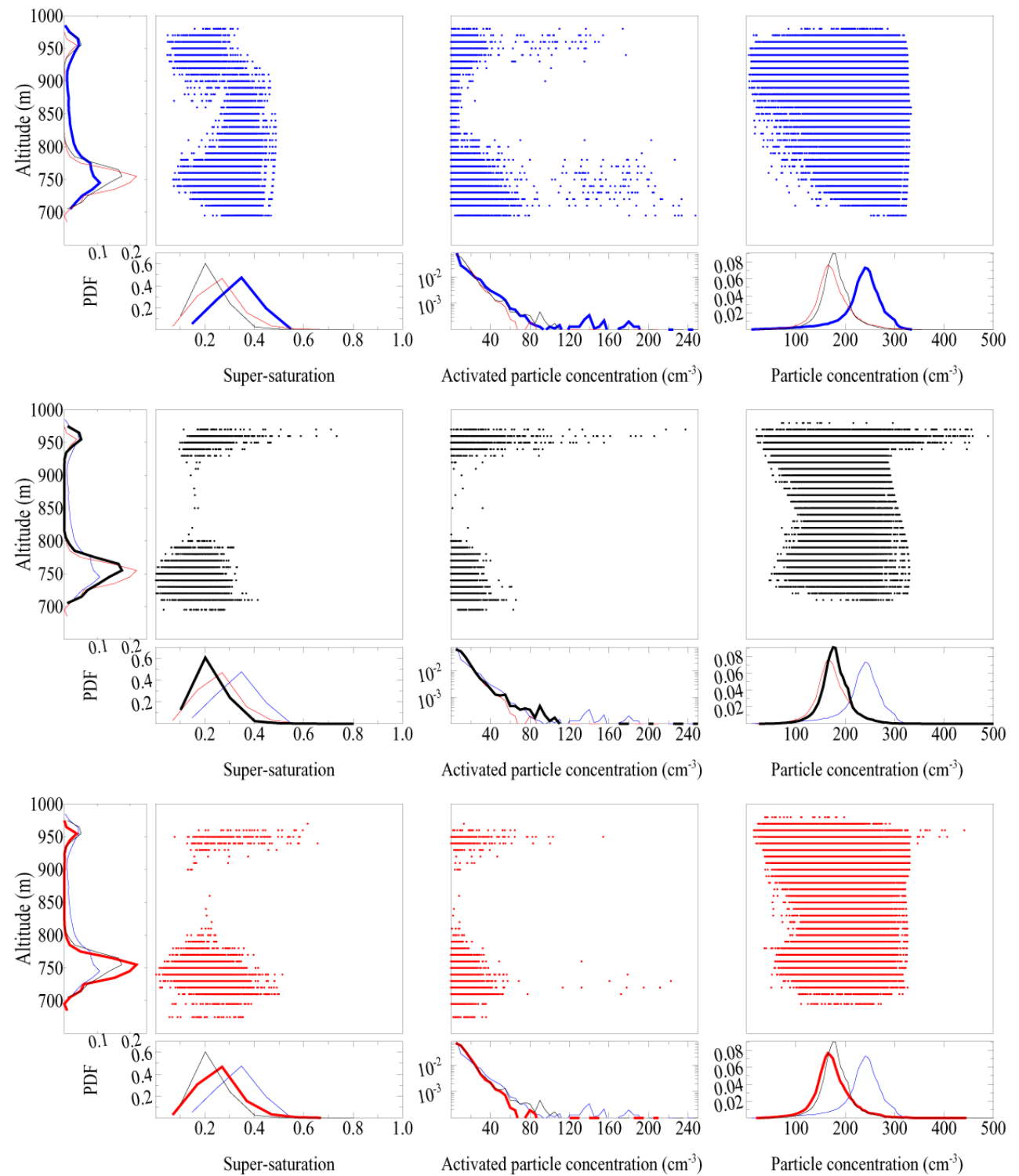

Fig. 3. For the DRY case: vertical distribution of supersaturation values in model grid boxes where CCN activation occurs (left), number concentration of activated particles, $N_{\mathrm{act}}$, during the time step (middle) and resulting number concentration of cloud droplets, $N_{\mathrm{c}}$, in all cloudy grid boxes (right) with the A (top-blue), B (middle line-black) and C (bottom-red) schemes. The far left graph shows the PDF of occurrence of activation events in the vertical, while the ones under each figure are for the PDF of parameter values.

In summary, this figure shows that, compared to the adjustment scheme (A), the diagnostic of supersaturation (B scheme) is efficient at simulating the progressive $\mathrm{CCN}$ activation at cloud base and the buffering of the supersaturation production in the cloud core due to existing LWC. It, however, reveals that the B scheme remains too sensitive to peak supersaturation artefacts, due to advection at the interface between cloudy and clear air, mainly at cloud top.

Figure 4 is similar to Fig. 3 for the WET case. As shown in Fig. 2, the WET case has a more homogeneous cloud fraction (the domain and layer cloud fraction LWC averages are sim- ilar from base to top) than the DRY case, suggesting that the moister entrained air produces less clear air patches within the cloud layer. As a result, the three schemes do not generate anymore $\mathrm{CCN}$ activation in the middle of the cloud layer, but the A scheme still significantly overestimates the cloud droplet number concentration at cloud base. Spurious CCN activation at cloud top is noticeable with the three schemes, although less pronounced with the $\mathrm{C}$ scheme. This figure illustrates the intrinsic limitations of an LES model (spatial and time resolutions) compared to Lagrangian simulations of the activation process, but still demonstrates that the 

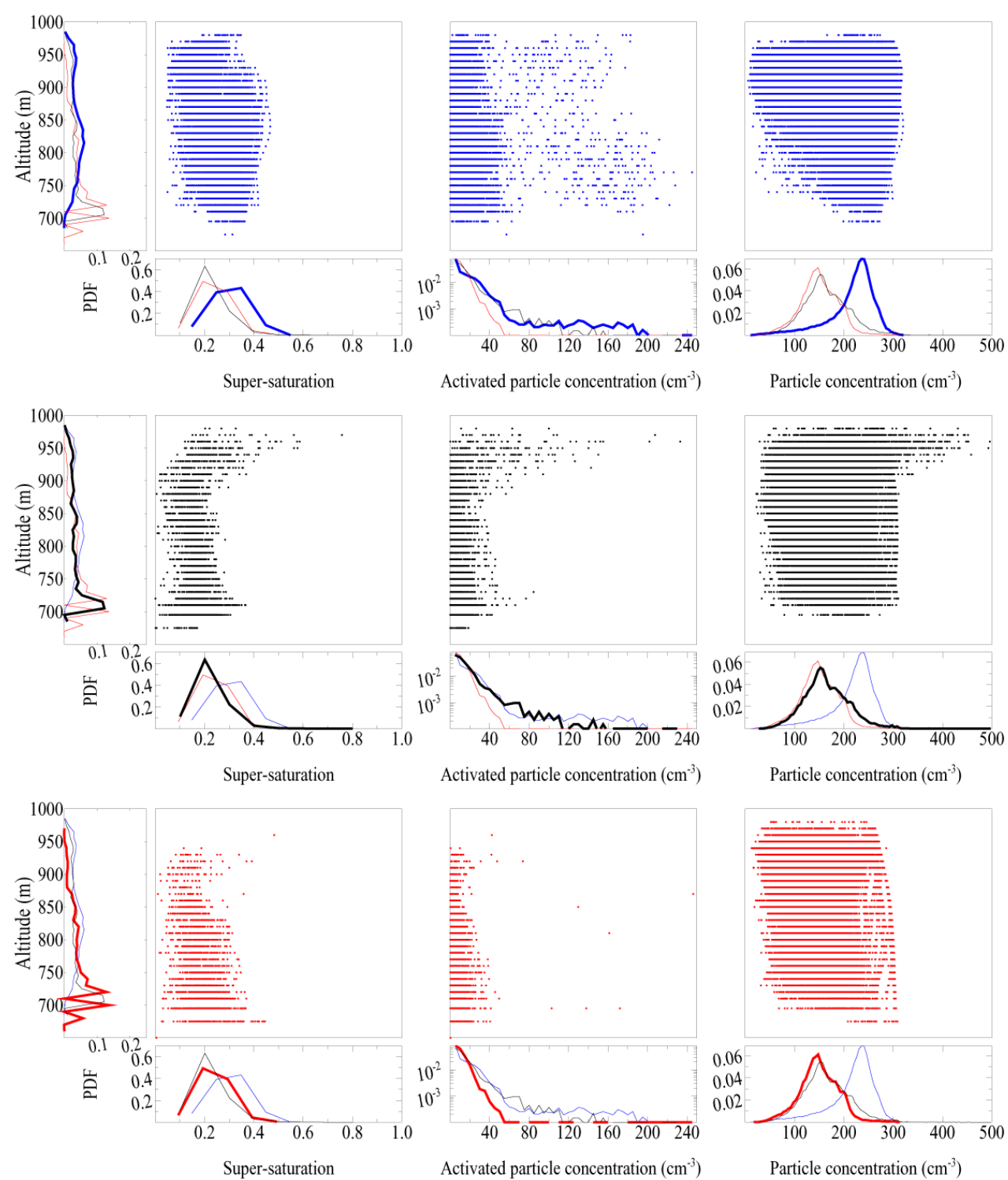

Fig. 4. Same as Fig. 3 for the WET case.

pseudo-prognostic approach efficiently mitigates the main activation artefacts of Eulerian models.

Figure 5 aims at illustrating how supersaturation is generated in a 3-D framework. For the A (top), B (middle) and $\mathrm{C}$ (bottom) schemes and the DRY (left) and WET (right) cases, the figure shows the supersaturation values in each grid box plotted against the vertical velocity. The PDF of the parameters is indicated on the left and under each graph. The colour scale corresponds to the liquid water mixing ratio in the grid box.

The top row directly reflects the A scheme parameterization of supersaturation as a function of vertical velocity that does not account for the presence of liquid water in the grid box. In contrast, both the middle and bottom rows attest that the $\mathrm{B}$ and $\mathrm{C}$ schemes only produce high values of supersaturation when the liquid water mixing ratio is reduced.

Interestingly, the $\mathrm{B}$ and $\mathrm{C}$ schemes generate extreme positive and negative values of supersaturation in grid boxes, with vertical velocities close to 0 . This feature also reflects the production of supersaturation when cloudy air is mixed with dry air (concavity of the Clausius-Clapeyron curve).

\subsection{Microphysical impact of entrainment-mixing}

The covariance of liquid water mixing ratio and cloud droplet number concentration provides an additional framework to further evaluate how realistic the simulations are. The technique has been extensively used to analyze in situ measurements of cloud microphysics (Burnet and Brenguier, 2007). 

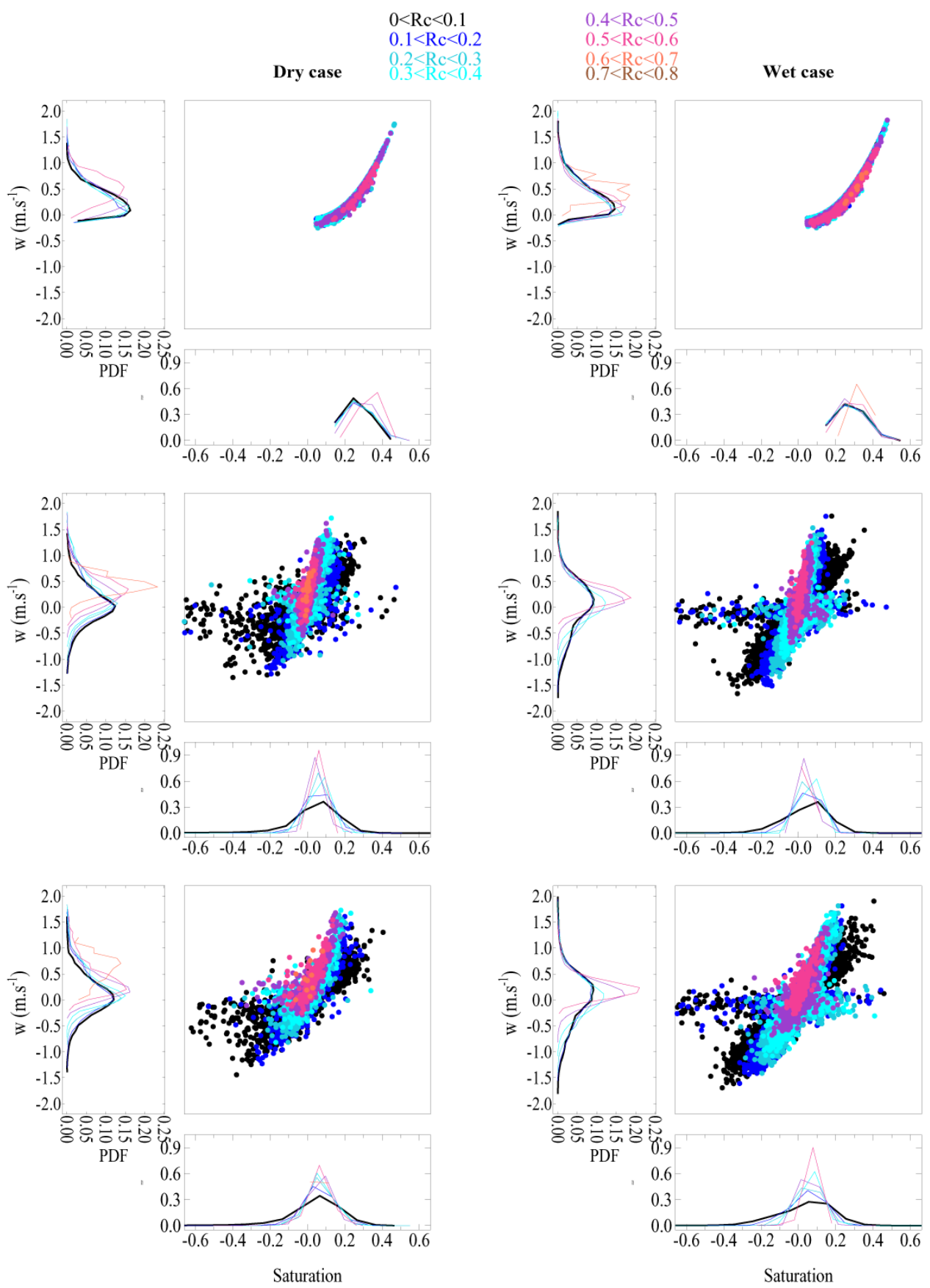

Fig. 5. Supersaturation as a function of vertical velocity for the A (top), B (middle) and C (bottom) schemes and the DRY (left) and WET (right) cases. The PDF of the parameters are indicated on the left and under each graph. The colour scale corresponds to the liquid water mixing ratio in the grid box.

Each measured sample is characterized by the cloud droplet number concentration and the mean volume radius (to the cube), $r_{\mathrm{v}}^{3}$, of the droplet size distribution:

$r_{\mathrm{v}}^{3}=\frac{3 \times q_{\mathrm{c}}}{4 \pi \rho_{\mathrm{w}} N_{\mathrm{c}}}$.
Both values are then normalised by their adiabatic values at the sample altitude level to correct the impact of fluctuations of the sample altitude above cloud base (see Burnet and Brenguier, 2007 for more details on the methodology). This methodology is illustrated in Fig. 6 with the grid box values from the upper $100 \mathrm{~m}$ of the cloud layer, as represented in Fig. 2 by the two green bars. The hyperbolas represent values 
of the LWC adiabatic ratio $\left(q_{\mathrm{c}} / q_{\mathrm{cad}}\right)$ from $1 \%$ for adiabatic samples (solid curve), down to $10 \%$ (dashed curves), and the thick dashed line in each panel shows the limit for pure homogeneous mixing. Indeed, when an adiabatic cloud volume is mixed with pure environmental air, characterized here by a water mixing ratio of 5 and $8 \mathrm{~g} \mathrm{~m}^{-3}$ for the DRY (left column) and WET (right) case, respectively, the results must lay to the left of the homogeneous mixing limit. The colour scale corresponds to the vertical velocity in the grid box. Obviously the adjustment scheme (top row) produces features that are different from the two other schemes and also different from in situ observations as reported in Burnet and Brenguier (2007). First, adiabatic values are grouped around the $1 / 1$ coordinates, while fluctuations of vertical velocity during CCN activation generally lead to a noticeable variability of the adiabatic cloud droplet number concentration. This is nicely simulated with the two other schemes (middle and bottom rows), as shown by the dispersion of the normalized values along the adiabatic LWC isoline (solid curve). This corroborates again the fact that the adjustment scheme activates everywhere in the cloud layer the same number concentration of CCNs, which corresponds to peak values of the supersaturation.

The most unrealistic feature, though, is the stratification of the cloud droplet number concentration dilution ratio $\left(N_{\mathrm{c}} / N_{\mathrm{ad}}\right)$ with the vertical velocity and the presence of superadiabatic droplet sizes (normalized droplet sizes greater than 1 on the Y-axis). These two features reflect the limitations of the adjustment scheme that only activate CCNs when the vertical velocity is positive. When cloudy air is mixed with dry air, the cloud droplet number concentration is diluted and supersaturation can be produced by the mixture of the two air masses, hence leading to activation of new CCNs; even the vertical velocity is close to 0 , or even negative. The adjustment scheme, which requires a positive velocity, is unable to activate new CCNs and condenses the supersaturated water vapour onto the remaining droplets, hence leading to super adiabatic growth.

For these two reasons, the adjustment scheme is not recommended for fine resolution LES simulations when the objective is to realistically simulate CCNs activation and entrainment-mixing processes impacts on cloud microphysics.

The $\mathrm{B}$ and $\mathrm{C}$ scheme show quite similar results, but one can notice the occurrence of superadiabatic cloud droplet number concentration (to the right of the homogeneous mixing limit) when using the B scheme (middle row). These unrealistic values of the cloud droplet number concentration after mixing are due to spurious $\mathrm{CCN}$ activation, already discussed in the previous section.

The $\mathrm{C}$ scheme is more robust with only very few cases of spurious $\mathrm{CCN}$ activation after mixing and realistic $N_{\mathrm{c}}, r_{\mathrm{v}}^{3}$ and vertical velocity distributions.

\subsection{Discussion}

These spurious values of supersaturation result in peak values of the droplet concentration, especially at cloud top, which are not noticeable from in situ measurements (Martin et al., 1994; Brenguier et al., 2000b; Pawlowska and Brenguier, 2003). We share the interpretation of Stevens et al. (1996a) that spurious activation at cloud top and edges is due to the still coarse spatial resolution of the model.

We agree with the interpretation of Grabowski and Morrison (2008), (denoted GM in the following) which suggests that this effect can be mitigated by adjusting state variables $T$ and $r_{\mathrm{v}}$ to the predicted supersaturation rather than using grid mean field of state variables $T$ and $r_{\mathrm{v}}$ to derive the supersaturation like proposed, for example, by Stevens et al. (1996b) (denoted SFCW in the following).

Scheme C, presented in this paper, is an intermediate between the approaches developed by SFCW and GM.

The dynamics forcing term (Eq. 8) is based on advection (including radiative transfer) of the thermodynamic variables to determine $S^{\prime}$. This is similar to SFCW, where the supersaturation is derived from advection of the thermodynamics.

The microphysics forcing term (Eq. 9) relies on $S^{t}$ and $r_{\mathrm{c}}$.

The supersaturation is then advanced in time from $S^{t}$, adding the two forcing terms above (see Eq. 10). Temperature and $r_{\mathrm{v}}$ are then adjusted to fit the computed supersaturation and $r_{\mathrm{c}}$. similarly to GM.

In summary, using scheme $\mathrm{C}$, the effects of spurious values of supersaturation are limited by the adjustment of the state variables $T$ and $r_{\mathrm{v}}$ to the predicted supersaturation, as suggested by GM. Unlike GM, our approach considers all types of forcings including radiative effects, while only the vertical velocity is considered in GM. This difference is crucial for the simulation of radiative fog. Indeed, in stratocumulus clouds most of the forcing comes from updraft, while in radiative fog formation, radiative forcing plays a key role.

\section{Conclusions}

Three parameterizations of supersaturation and $\mathrm{CCN}$ activation have been tested here in the framework of a 3-D LES model that aims at predicting both LWC and the cloud droplet number concentration in convective clouds.

The first one assumes no supersaturation and the liquid water mixing ratio is exactly equal to the difference between the total mixing ratio and the saturated one. For $\mathrm{CCN}$ activation, a peak value of supersaturation is diagnosed at each time step based on a formula that involves the vertical velocity in the grid box as a forcing term and a parameterized description of the $\mathrm{CCN}$ activation spectrum, assuming (i) the conditions last long enough for the supersaturation to reach its maximum and (ii) the grid box is initially void of droplets.

The various tests demonstrate that such a parameterization is not effective when the vertical resolution and the 

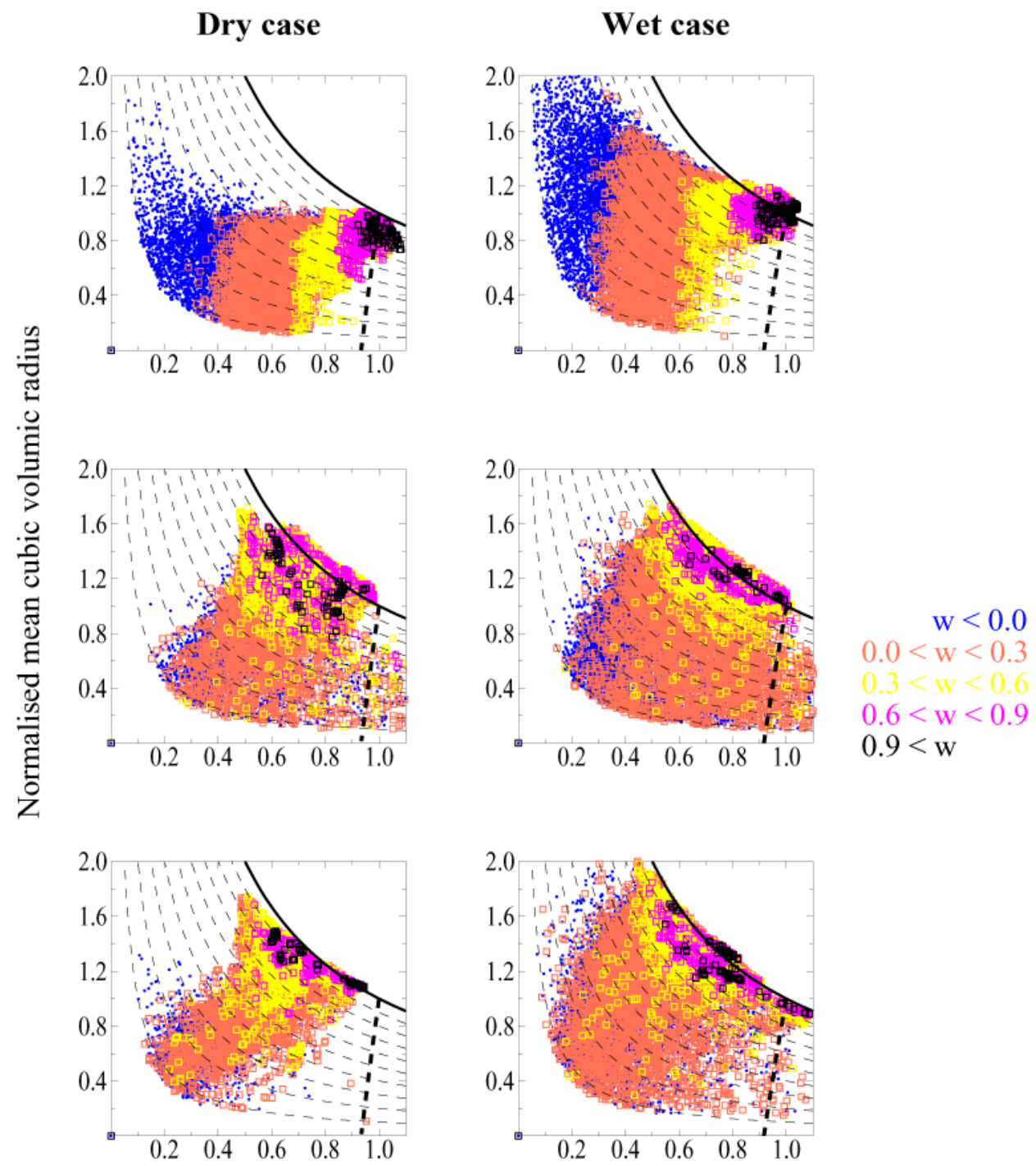

Normalised mean cloud droplet number concentration

Fig. 6. Normalised mean cubic volume radius as a function of the normalised mean cloud droplet number concentration from the upper $100 \mathrm{~m}$ of the cloud layer for the DRY (left) and the WET (right) cases. Solid and dashed lines represent values of the LWC dilution ratio $\left(q_{\mathrm{c}} / q_{\mathrm{cad}}\right)$ from $1 \%$ for adiabatic samples (solid curve), down to $10 \%$ (dashed curves). Thick dashed line shows the limit for pure homogeneous mixing. The colour scale corresponds to the vertical velocity in the grid box.

time steps are much shorter than what is necessary for (i) to be valid. Moreover, (ii) is obviously not fulfilled within the cloud core after CCN activation is completed and droplets have grown enough. Consequently, the scheme keeps activating new CCNs in the cloud core, while explicit calculations of the supersaturation would suggest the opposite. Finally, at cloud top, the scheme is unable to diagnose production of supersaturation by mixing because the vertical velocity is mostly null or negative. Consequently, it condenses the surplus of water vapour onto a diluted population of droplets that progressively develop superadiabatic unrealistic sizes.
The second scheme involves an additional variable to keep track of the condensed water. An intermediate value of supersaturation is diagnosed from the temperature, total and liquid water mixing ratios after advection and processes other than microphysics. This value is then used for $\mathrm{CCN}$ activation and condensational droplet growth. Because condensed water is accounted for in the diagnostic of supersaturation, this scheme avoids the main limitations of the previous one and it realistically simulates the variability of cloud droplet number concentration due to the variability of vertical velocity during the activation process. It also simulates supersaturation production by mixing at cloud top, but it is slightly too 
sensitive to small errors in the advection scheme and significantly overestimates $\mathrm{CCN}$ activation at the top.

The third scheme builds up upon the benefits of the second one, but also adds a pseudo-prognostic of the supersaturation, based on approximations of the source and sink terms. Introducing the dependence on the time step by integrating the time evolution of supersaturation, this scheme smoothes out spurious fluctuations of temperature and water mixing ratios, hence of the supersaturation and produces more realistic values of the cloud droplet number concentration at cloud top.

It is therefore recommended to use such an improved scheme when the spatial and time resolutions of the model are shorter than $50 \mathrm{~m}$ and $10 \mathrm{~s}$, respectively, and when the objective is to realistically simulate $\mathrm{CCN}$ activation droplet growth and the impact of mixing. Note, however, that mixing is assumed to be homogeneous and the scheme is unable to replicate the inhomogeneous mixing features that are currently observed in stratocumulus clouds.

\section{Appendix A}

\section{Condensation/evaporation forcing term for the supersaturation}

The equation for the time evolution of the supersaturation is derived from Eq. (2):

$$
\frac{d S}{\mathrm{~d} t}=\frac{1}{r_{\mathrm{s}}} \frac{\mathrm{d} r_{\mathrm{v}}}{\mathrm{d} t}-\frac{(S+1)}{r_{\mathrm{s}}} \frac{\mathrm{d} r_{\mathrm{s}}}{\mathrm{d} t}
$$

When only water phase changes are considered,

$$
\frac{\mathrm{d} r_{\mathrm{v}}}{\mathrm{d} t}=-\frac{\mathrm{d} r_{\mathrm{c}}}{\mathrm{d} t}
$$

and

$$
\frac{\mathrm{d} r_{\mathrm{s}}}{\mathrm{d} t}=\frac{\mathrm{d} r_{\mathrm{s}}}{\mathrm{d} T} \frac{\mathrm{d} T}{\mathrm{~d} t}=\frac{\mathrm{d} r_{\mathrm{s}}}{\mathrm{d} T} \frac{L_{\mathrm{v}}}{C_{\mathrm{p}}} \frac{\mathrm{d} r_{\mathrm{c}}}{\mathrm{d} t}
$$

where $L_{\mathrm{v}}$ and $C_{\mathrm{p}}$ are the latent heat of condensation and the specific heat at constant pressure.

Equation (A3) leads to Eq. (9).

Acknowledgements. This work has been partly funded by EUCAARI (European Integrated project on Aerosol Cloud Climate and Air Quality interactions) No. 036833-2.

Edited by: K. Gierens

\section{References}

Abdul-Razzak, H. and Ghan, S. J.: A parameterization of aerosol activation 2, multiple aerosol types, J. Geophys. Res., 105, 6837 6844, 2000.

Brenguier, J. L., Chang, P. Y., Fouquart, Y., Johnson, D. W, Parol, F., Pawlowska, H., Pelon, J., Schueller, F., and Snider, J.: An overview of the ACE-2 CLOUDYCLOUMN closure experiment, Tellus B, 52, 815-927, 2000a.

Brenguier, J. L., Pawlowska, H., Schueller, F., Reusker, R., Fischer, J., and Fouquart, Y: Radiative properties of boundary layer clouds: droplet effective radius versus number concentration, J. Atmos. Sci., 57, 803-821, 2000b.

Burnet, F. and Brenguier, J. L.: Observational study of the entrainment mixing process in warm convective clouds, J. Atmos. Sci., 64, 1995-2011, 2007.

Charnock, H.: Wind stress over a water surface, Q. J. Roy. Meteor. Soc., 81, 639-640, 1955.

Chosson, F., Brenguier, J. L., and Schüller, L.: Entrainment mixing and radiative transfer simulation in boundary layer clouds, $\mathbf{J}$ Atmos. Sci., 64, 2670-2682, 2007.

Clark, T. L.: Numerical modeling of the dynamics and microphysics of warm cumulus convection, J. Atmos. Sci., 30, 857-878, 1973.

Cohard, J. M., Pinty, J., and Bedos, C.: Extending twomey's analitical estimate of nucleated cloud droplet concentration from ccn spectra, J. Atmos. Sci, 55, 3348-3357, 1998.

Cohard, J. M., Pinty, J., and Suhre, K.: On the parameterization of activation spectra from cloud condensation nuclei microphysical properties, J. Geophys. Res., 105, 11753-11766, 2000.

Cuxart, J., Bougeault, P., and Redelsperger, J. L.: A turbulence scheme allowing for mesosacle and large eddy simulation, Q. J. Roy. Meteor. Soc., 126, 1-30, 2000.

De Roode, S. R., Duynkerke, P. G., and Jonker, H. J. J: Large-Eddy Simulation: How large is large enough?, J. Atmos. Sci., 61, 403421, 2004

Ghan, S. J., Chuang, C. C., Easter, R. C., and Penner, J. E.: A parameterization of cloud droplet nucleation, Part II: Multiple aerosol types, Atmos. Res., 36, 39-54, 1995.

Geoffroy, O., Brenguier, J.-L., and Sandu, I.: Relationship between drizzle rate, liquid water path and droplet concentration at the scale of a stratocumulus cloud system, Atmos. Chem. Phys., 8, 4641-4654, doi:10.5194/acp-8-4641-2008, 2008.

Grabowski, W. W.: Numerical experiments on the dynamics of the cloud-environment interface: Small cumulus in a shearfree environment, J. Atmos. Sci., 46, 3513-3541, 1989.

Grabowski, W. W. and Morrison, H.: Toward the mitigation of spurious cloud-edge supersaturation in cloud models, Mon. Weather Rev., 136, 1224-1234, 2008.

Grabowski, W. W. and Smolarkiewicz, P. K.: Monotone finite difference approximations to the advection-condensation problem, Mon. Weather Rev., 118, 2082-2097, 1990.

Khairoutdinov, M. and Kogan, Y.: A new cloud physics parameterization in a large-eddy simulation model of marine stratocumulus, Mon. Weather Rev., 128, 229-243, 2000.

Klaassen, G. P. and Clark, T. L.: Dynamics of the cloud environment interface and entrainment in small cumuli: Twodimensional simulations in the absence of ambient shear, J. Atmos. Sci., 42, 2621-2642, 1985.

Kogan, Y. L., Khairoutdinov, M. P., Lilly, D. K., Kogan, Z. N., and Liu, Q.: Modeling of stratocumulus cloud layers in a large eddy 
simulation model with explicit microphysics, J. Atmos. Sci., 52, 2923-2940, 1995.

Lafore, J. P., Stein, J., Asencio, N., Bougeault, P., Ducrocq, V., Duron, J., Fischer, C., Héreil, P., Mascart, P., Masson, V., Pinty, J. P., Redelsperger, J. L., Richard, E., and Vilà-Guerau de Arellano, J.: The Meso-NH Atmospheric Simulation System. Part I: adiabatic formulation and control simulations, Ann. Geophys., 16, 90-109, doi:10.1007/s00585-997-0090-6, 1998.98.

Leaitch, W. R., Strapp, J. W., Isaac, G. A., and Hudson, J. G.: Cloud droplet nucleation and cloud scavenging of aerosol sulphate in polluted atmopheres, Tellus B, 38, 328-344, 1986.

Martin, G. M., Johson, D. W., and Spice, A.: The measurement and parameterization of effective radius of droplets in warm stratocumulus clouds, J. Atmos. Sci., 51, 1823-1842, 1994.

Morcrette, J. J.: Radiation and cloud radiative properties in the ECMWF operational weather forecast model, J. Geophys. Res., 96D, 9121-9132, 1991.

Pawlowska, H. and Brenguier, J. L.: An observationnal study of drizzle formation in stratocumulus clouds for general circulation model (GCM) parameterizations, J. Geophys. Res., 108, 8630, doi:10.1029/2002JD002679, 2003.
Press, W. H., Teukolsky, S. A., Vetterling, W. T., and Flannery, B. P.: Numerical Recipes in FORTRAN: The art of scientific computing, 2nd Edn., Cambridge University Press, 963 pp., 1992.

Pruppacher, H. R. and Klett, J. D.: Microphysics of Clouds and Precipitation, Kluwer Academic, 954 pp., 1997.

Sandu, I., Brenguier, J. L., Geoffroy, O., Thouron, O., and Masson, V.: Aerosol impacts on the diurnal cycle of marine stratocumulus, J. Atmos. Sci., 65, 2705-2718, 2008.

Sandu, I., Brenguier, J.-L., Thouron, O., and Stevens, B.: How important is the vertical structure for the representation of aerosol impacts on the diurnal cycle of marine stratocumulus?, Atmos. Chem. Phys., 9, 4039-4052, doi:10.5194/acp-9-4039-2009, 2009.

Stevens, B., Walko, R. L., and Cotton, W. R.: The spurious production of cloud edge supersaturation by eulerian models, Mon. Weather Rev., 124, 1034-1035, 1996a.

Stevens, B., Feingold, G., Cotton, W. R., and Walko, R. L.: Elements of the microphysical structure of numerically simulated nonprecipitating stratocumulus, J. Atmos. Sci., 53, 980-1006, $1996 b$.

Twomey, S.: The nuclei of natural cloud formation, Part II: The supersaturation in natural clouds and the variation of cloud droplet concentration, Pure Appl. Geophys., 43, 243-249, 1959. 